\title{
Conductance of Sodium Nitrate in Methanol -Water Mixtures at Different Temperatures
}

\author{
Ajaya Bhattarai ${ }^{1}$, Deepak Sapkota ${ }^{2}$, Netra P. Subedi ${ }^{2}$ \\ Manoj Khanal $^{2}$, Tulsi P. Niraula ${ }^{1}$ \\ ${ }^{1}$ Mahendra Morang Adarsh Multiple Campus, Tribhuvan University, Biratnagar \\ ${ }^{2}$ Central Campus of Technology Tribhuvan University Hattisar, Dharan \\ e-mail: bkajaya@yahoo.com
}

\begin{abstract}
Precise measurements on the conductivity of Sodium nitrate in methanol-water mixed solvent media containing $0.10,0.20,0.30$ and 0.40 volume fractions of methanol at 308.15, 318.15 and $323.15 \mathrm{~K}$ are reported. The concentrations were varied from (0.001 to 0.15$)$ mol. $\mathrm{L}^{-1}$. The results showed a sharp increase in the conductivity with increasing electrolyte concentration. Also, the conductivity of electrolyte increases with increase in temperature whereas the conductivity of electrolyte decreases with increase in the amount of methanol.
\end{abstract}

Key words: conductivity, sodium nitrate, relative permittivity, mixed solvent media

\section{Introduction}

From the concentration dependence of the electrolyte conductivity, different quantities strongly influenced by solvent properties can be derived. Their change with mixed-solvent composition may thus reflect the change in solvent structure and ion-solvent interactions. A binary mixture of water and some organic solvent, with their ratio varying in a wide range, is the most frequently investigated medium (Sokol et al .2006).

Although numerous conductance measurements have been reported in the literature, such studies in methanol - water mixed solvents are relatively rare (Janz et al. 1972). Methanol water mixtures have very special properties, which are different from that of the other alcohol + water mixtures. Ion association has been found to be negligible upto a methanol content of about $80 \%$ in the binary solvent. It may be due to the larger dielectric constant of the methanol-water mixtures and the smaller ion size. The transport properties have been investigated for a wide variety of electrolytes in methanol - water mixed solvent media in great detail (Shedlovsky \& Kay 1956, Kubota \& Masatoki 1976, Zhang et al. 1996, 1997, Kubota \& Horimoto 1999; Chatterjee \& Das 2006).

Earlier, the transport properties have been investigated (Bhattarai et al. 2006) for a polyelectrolyte in methanolwater mixed solvent media. The experimental data was analyzed by Manning Model and found the lower values than the experimental ones. Later on (Bhattarai, 2008) used the Scaling Theory Approach and obtained good fitting with the experimental data. It is our interest to see the effects of concentration, relative permittivity and temperature on the transport properties of Sodium nitrate in methanol-water mixed solvent media. 


\section{Methodology}

Methanol (Merck, India) was distilled with phosphorous pentoxide and then redistilled over calcium hydride. The purified solvent had a density of $0.77723 \pm 0.00004$ g.cm ${ }^{-3}$ which was measured by the use of an Ostwald-Sprengel type pycnometer of about $25 \mathrm{~cm}^{3}$ capacity. The solvent was transfused into the pycnometer by using a medical syringe. The pycnometer was then tightly fixed in a thermostat at the experimental temperatures within $0.005 \mathrm{~K}$. After thermal equilibrium was attained, the mass of the pycnometer was measured with an electronic balance, and the density was calculated. Density measurements are precise within $0.00005 \mathrm{~g} . \mathrm{cm}^{-3}$, which is satisfactory for our purpose. A co-efficient of viscosity of 0.47424 $\pm 0.00005 \mathrm{mPa}$.s was determined by the viscometric measurements at $308.15 \mathrm{~K}$ a Schultz-Immergut-type viscometer (Schulz Immergut 1952) with a sintered disc fitted to the widest arm to filter the solution/ solvent from dust particles, if any and these values are in good agreement with the literature values (Moumouzias et al. 1991). Triply distilled water with a specific conductance less than $10^{-6}$ S.cm ${ }^{-1}$ at $308.15 \mathrm{~K}$ was used for the preparation of the mixed solvents. The physical properties of methanol-water mixed solvents used in this study at 308.15, 318.15, and 323.15K are shown in Table 1 . The relative permittivity of methanol-water mixtures at the experimental temperatures were obtained by regressing the relative permittivity data as function of solvent composition from the literature (Albright \& Gasting 1946).

Table 1. Properties of Methanol-Water Mixtures Containing 0.1, 0.2, 0.3, and 0.4 volume fractions of Methanol at $(308.15,318.15$, and 323.15) K.

\begin{tabular}{cccc}
\hline $\mathrm{T}^{*} / K$ & $\rho_{0}{ }^{*} / \mathrm{g} \cdot \mathrm{cm}^{-3}$ & $\eta_{0}{ }^{*} / \mathrm{mPa} . \mathrm{s}$ & $D^{*}$ \\
\hline & 0.1 volume fractions of methanol & \\
308.15 & 0.9797 & 0.8665 & 71.57 \\
323.15 & 0.9760 & 0.7017 & 68.18 \\
& 0.9741 & 0.6375 & 66.45 \\
308.15 & 0.2 volume fractions of methanol & \\
318.15 & 0.9663 & 1.0217 & 68.14 \\
323.15 & 0.9616 & 0.8075 & 64.80 \\
& 0.9588 & 0.7300 & 63.15 \\
308.15 & 0.3 volume fractions of methanol & \\
318.15 & 0.9516 & 1.1418 & 64.25 \\
323.15 & 0.9463 & 0.8957 & 60.99 \\
& 0.9433 & 0.8052 & 59.41 \\
308.15 & 0.4 volume fractions of methanol & \\
318.15 & 0.9310 & 1.2034 & 60.34 \\
323.15 & 0.9254 & 0.9309 & 57.18 \\
& 0.9234 & 0.8288 & 55.62 \\
\hline
\end{tabular}

"Chatterjee and Das 2006, Bhattarai et al 2006

Sodium nitrate $\left(\mathrm{NaNO}_{3}\right)$ employed in these investigations was purchased from Ranbaxy Chemical Company, Inc., India. Conductance measurements were carried out on a Pye-Unicam PW 9509 conductivity meter at a frequency of $2000 \mathrm{~Hz}$ using a dip-type cell with a cell constant of $1.15 \mathrm{~cm}^{-1}$ and having an uncertainty of $0.01 \%$. The cell was calibrated by the method of Lind and co-workers, 1959 using aqueous potassium chloride solution. The measurements were made in a water bath maintained within $\pm 0.005 \mathrm{~K}$ of the desired temperature. The details of the experimental procedure have been described earlier (Das \& Hazra 1992, 1995). Several independent solutions were prepared and runs were performed to ensure the reproducibility of the results. Due correction was made for the specific conductance of the solvent by subtracting the specific conductance of the relevant solvent medium from those of the electrolyte solutions.

In order to avoid moisture pickup, all solutions were prepared in a dehumidified room with utmost care. In all cases, the experiments were performed in three replicates. 


\section{Results and Discussion}

The experimental specific conductivities of sodium nitrate as a function of the salt concentration $\left(c_{\mathrm{s}}\right)$ at 308.15, 318.15 and $323.15 \mathrm{~K}$ of four different methanolwater mixtures (containing 0.10, 0.20, 0.30 and 0.40 volume fractions of methanol) are depicted in figures
1-3. From these figures, it is evident that the specific conductivities exhibit a sharp increase with increasing concentration within the concentration range investigated here. The increase in the conductance with concentration is due to an increase in the number of ions per unit volume of the solution.

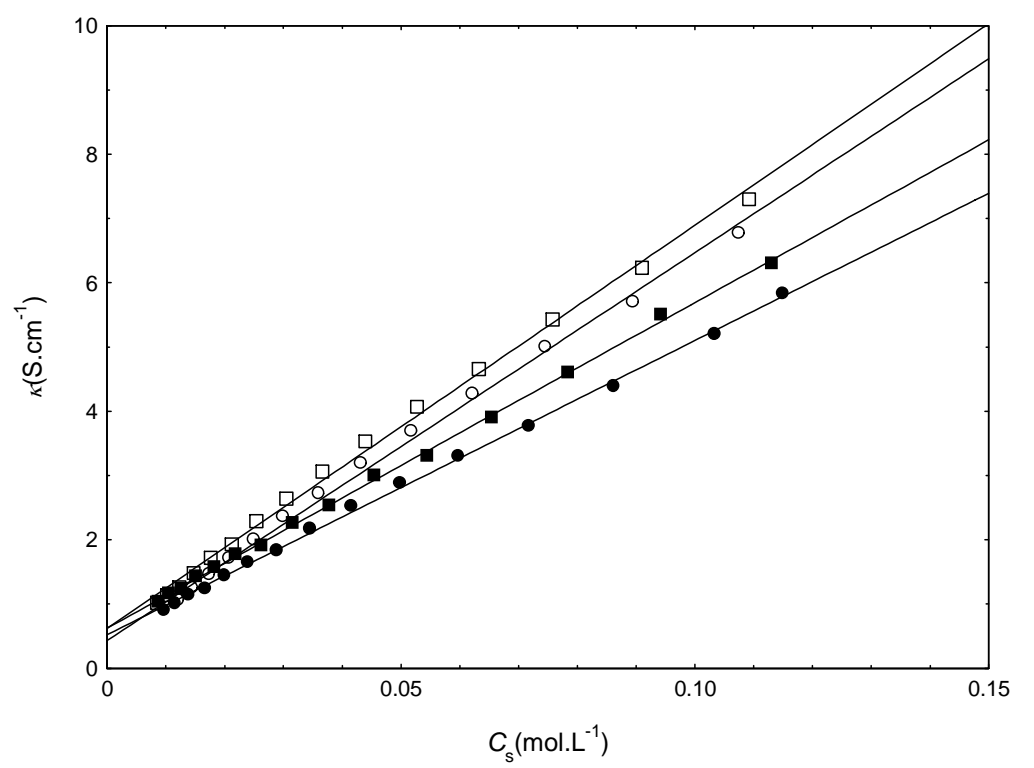

Fig. 1. Specific conductivities of $\mathrm{NaNO}_{3}$ as a function of the salt concentration $\left(c_{\mathrm{s}}\right)$ in $308.15 \mathrm{~K}$ : open squares, open circles, closed squares and closed circles represent $0.10,0.20,0.30$ and 0.40 volume fractions of methanol in the solvent mixture respectively.

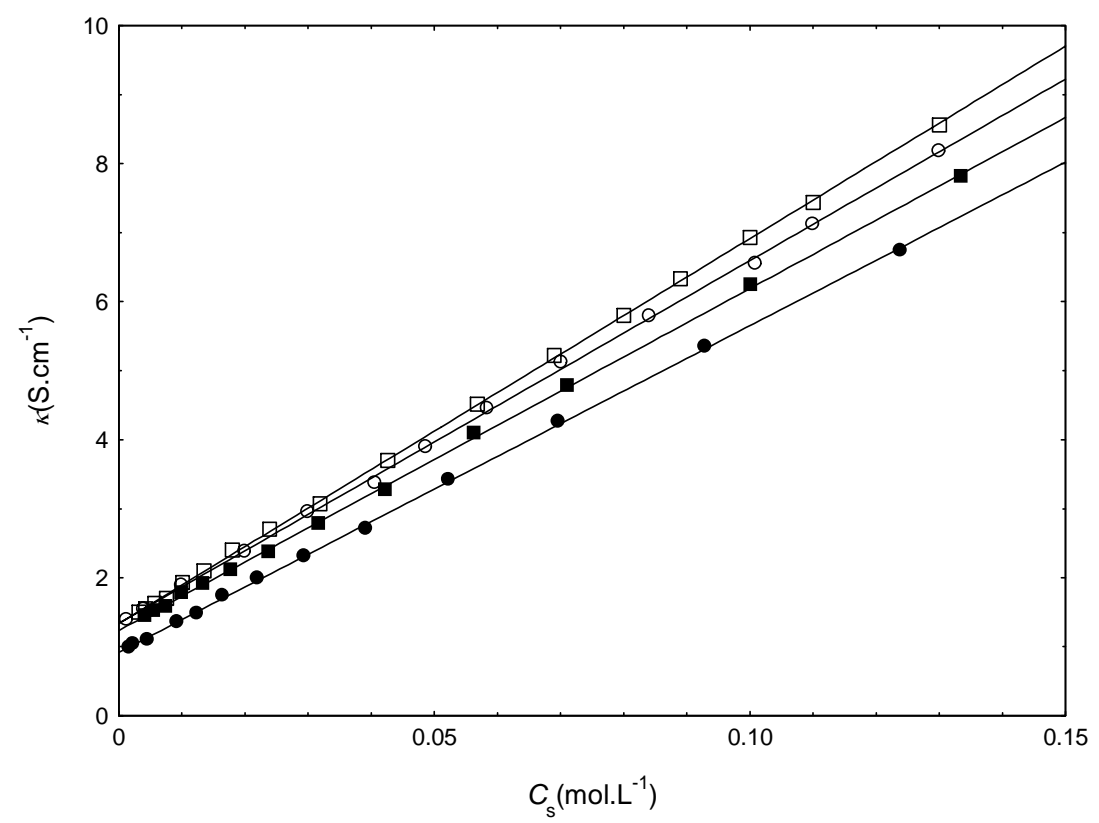

Fig. 2. Specific conductivities of $\mathrm{NaNO}_{3}$ as a function of the salt concentration $\left(c_{\mathrm{s}}\right)$ in $318.15 \mathrm{~K}$ : open squares, open circles, closed squares and closed circles represent $0.10,0.20,0.30$ and 0.40 volume fractions of methanol in the solvent mixture respectively. 


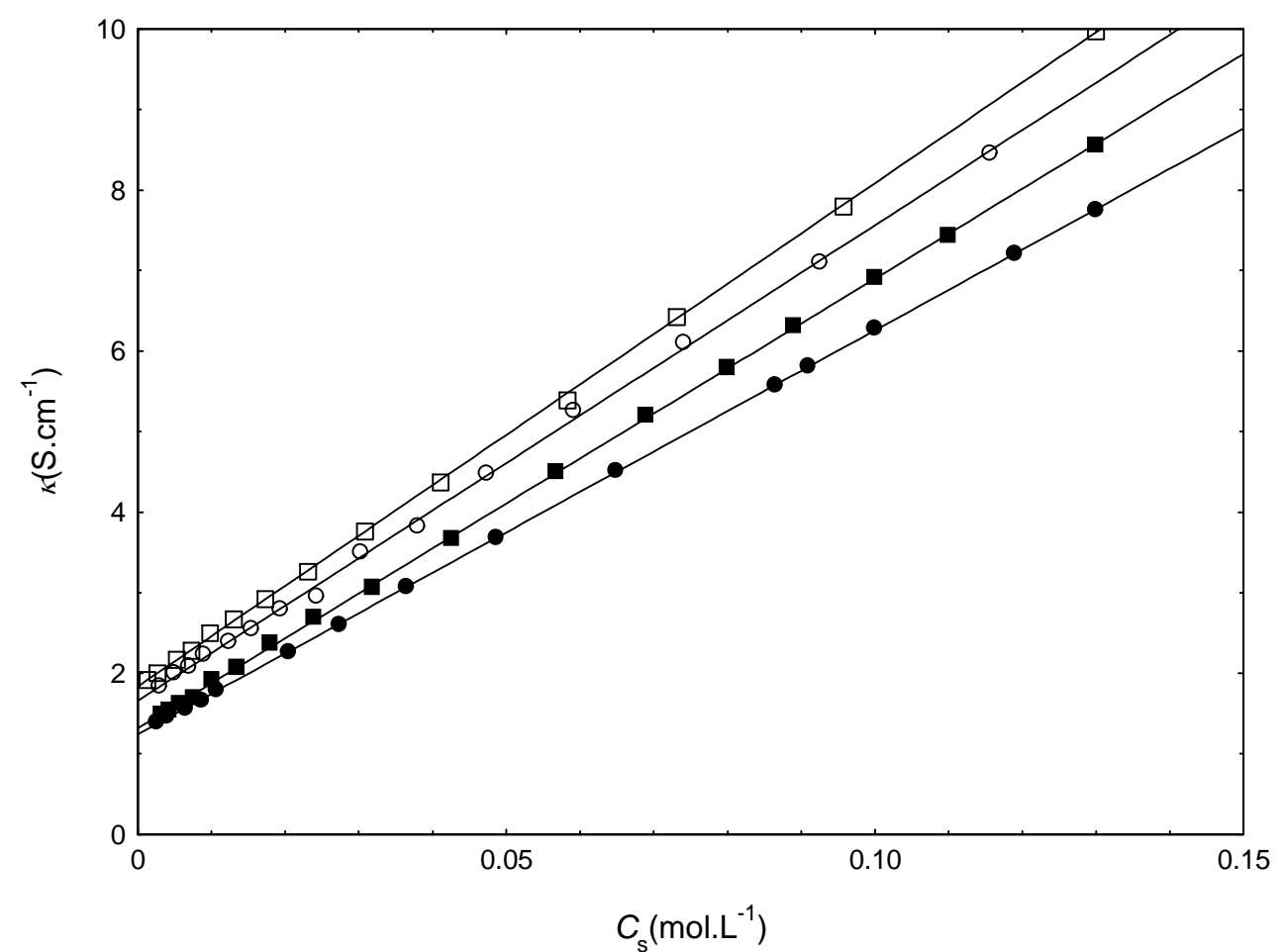

Fig. 3. Specific conductivities of $\mathrm{NaNO}_{3}$ as a function of the salt concentration $\left(c_{\mathrm{s}}\right)$ in $323.15 \mathrm{~K}$ : open squares, open circles, closed squares and closed circles represent $0.10,0.20,0.30$ and 0.40 volume fractions of methanol in the solvent mixture respectively.

Obviously, the concentration dependence of the specific conductivity follows the same pattern at all the temperatures and solvent composition investigated. It has been studied earlier that the conductivity value decreases with increase of methanol in the system (Bhattarai et al. 2006). The conductance decreases with increase of alcohol content for the studied methanol-water mixed solvent system. The presence of methanol reduces the dielectric constant of the solvent phase and makes easier for the formation of ion-pairs in the solution phase. In other words, in solvents of low dielectric constants, having small ionizing effect on the electrolytes, the electrostatic forces between oppositely charged ions would be appreciable and conductance value will have small value. However, solvents with high dielectric constants yield more conducting solutions.

The conductivity of an electrolyte depends upon the temperature. With increase in temperature, the conductivity of an electrolyte increases. Here, the conductivity of sodium nitrate increases with increasing temperatures in methanol - water mixed solvent media which was also seen in the system (Bhattarai et al. 2006). The rise in conductance with temperature is due to the decrease in the viscosity of the solution, increase in the speed of the ions and an increase in the degree of ionization.

\section{Acknowledgements}

One of the authors (Deepak Sapkota) is thankful to University Grant Commission (UGC), Nepal, for providing financial support to pursue the research under Mini-Research Grant for the fiscal year 2010/ 2011. Sincere thanks to the department of chemistry, Central Technology Campus (Tribhuvan University), Hattisar, Dharan, Nepal and head of department of chemistry, Mahendra Morang Adarsh Multiple Campus, Biratnagar, Tribhuvan University, Nepal for providing the available research facilities to conduct this research work.

\section{References}

Albright, P. S. and L. J. Gasting. 1946. Dilectric Constants of Methanol-Water System from 5 to $55^{\circ}$. J. Am. Chem. Soc. 68: 1061-1063. 
Ajaya Bhattarai et al./Conductance of Sodium

Bhattarai, A., P. Nandi and B. Das. 2006. The Effects of Concentration, Relative Permittivity and Temperature on the Transport Properties of Sodium Polystyrenesulphonate in Methanol-Water Mixed Solvent Media. J. Pol. Res. 13: 475-482.

Bhattarai, A. 2008. Electrical Conductivity of Semidilute Solution of Sodium Polystyrenesulfonate in MethanolWater Mixtures at Four Different Temperatures: The Scaling Theory Approach. Nepal Journal of Science and Technology 9: 163-170.

Chatterjee, A. and B. Das. 2006. Electrical Conductances of Tetrabutylammonium Bromide, SodiumTetraphenylborate, and Sodium Bromide in Methanol (1) + Water (2) Mixtures at (298.15, 308.15, and 318.15) K. J. Chem. Eng. Data, 51: 1352-1355.

Das, B. and D. K. Hazra. 1992. Studies on the Viscosities, Conductances, and Adiabatic Compressibilities of Some Tetraalkylammonium Perchlorates in 2-Methoxyethanol. Bull. Chem. Soc. Jpn. 65: 3470-3476.

Das, B. and D. K. Hazra. 1995. Conductometric, Viscometric, and Spectroscopic Investigations on the Solvation Phenomena of Alkali-Metal Ions and Ion pairs in 2Methoxyethanol. J. Phys. Chem. 99: 269-273.

Janz, G.T. and R.P.T. Tomkins. 1972. Non Aq. Electro. Handbook; Academic Press: London, Vol.1.

Kubota, E. and S. Horimoto. 1999. Conductivity measurements of alkali metal thiocyanates in watermethanol mixtures. Nippon Kagaku Kaishi, 203-206.
Kubota, E. and M. Yokoi. 1976. Conductance of some High valence type electrolytes in mixed solvents. I. Conductance of Tris(1,10-phenanthroline)iron(II) sulfate and Chloride in water-Methanol Mixtures at 298.15K. Bulletin of the Chemical Society of Japan, 49(10): 2674-2678.

Lind, J. E., Jr., J. J. Zwolenik and R.M. Fuoss. 1959. Calibration of Conductance Cells at $25^{\circ} \mathrm{C}$ with Aqueous Solutions of Potassium Chloride. J. Am. Chem.Soc. 81: 1557-1559.

Moumouzias, G. D., K. Panopoulos, and G. Ritzoulis. 1991. Excess properties of the binary liquid system propylene carbonate + acetonitrile. J. Chem. Eng. Data, 36: 20-23.

Schulz, J. and E. H. Immergut. 1952. Instrument for determining shear dependence of intrinsic viscosity of high polymer solutions. J. Polym. Sci., 9: 279-281.

Shedlovsky, T. and R. L. Kay. 1956. The ionization constant of Acetic Acid in water-methanol mixtures at 298.15K form Conductance measurements. J. Phys. Chem., 60: $151-155$.

Sokol, V., R. Tomas, M. Visic and I. Tominic. 2006. Conductometric Study of Potassium Bromide in 2Butanol +Water Mixtures. J. Solution Chem. 35: 16871698.

Zhang, S., S. Han and Y. Jin. 1996. Molar Conductance of $\mathrm{NaCl}$ and $\mathrm{KCl}$ in Aqueous Methanol Mixtures at 298.15K. Acta Physico- Chemica Sinica, 12(1): 75-80.

Zhang, S., H. Li, S. Dai, T. Wang, and S. Han. 1997. Molar Conductances of Sodium Bromide and Sodium Iodide in Methanol + Water at 298.15 K. J. Chem. Eng. Data, 42: 651-654. 
Nepal Journal of Science and Technology 12 (2011) 187-192 